\title{
Tratamiento ortodóntico compensatorio en patrón esquelético clase III mediante el uso del arco MEAW
}

\author{
Compensatory orthodontic treatment of skeletal class III using the \\ MEAW arch
}

Daisy Barazorda Gonzales ${ }^{\text {1a }}$

ID https://orcid.org/0000-0002-4411-7492

${ }^{1}$ Investigador independiente.

${ }^{a}$ Cirujano Dentista.

\section{Resumen}

El objetivo de este reporte de caso fue evaluar el efecto de la técnica MEAW (Multiloop Edgewise Arch Wire) en el arco mandibular como parte del tratamiento compensatorio de un paciente con patrón esquelético de clase III; para tal fin, se tomaron radiografías cefalométricas laterales; las cuales fueron analizadas antes y después del tratamiento. En el presente caso se obtuvo una oclusión de clase I, logrando al final del tratamiento un overjet y overbite adecuados; además, se estableció la forma parabólica, tanto en el arco superior como en el inferior, con lo cual se redujo la distancia intercanina en $2 \mathrm{~mm}$. Se concluyó que la técnica MEAW con un solo arco, combinada con elásticos de clase III, puede corregir la maloclusión de clase III esquelética. La técnica MEAW y los elásticos de clase III modificados proporcionaron una estrategia de tratamiento adecuado, especialmente para pacientes con un ángulo alto y una tendencia a la mordida abierta.

Palabras clave: Técnica MEAW, patrón esquelético clase III.

\begin{abstract}
The objective of this case report was to evaluate the effect of the MEAW technique (Multiloop Edgewise Archwire) on the mandibular arch in compensatory treatment of a patient with a class III skeletal pattern. Lateral cephalometric radiographs were taken, which were analyzed before and after treatment. In the present case, a class I occlusion was obtained, achieving an adequate Overjet and Overbite; and the parabolic shape of the upper and lower arch was established, thus reducing the intercanine distance by $2 \mathrm{~mm}$. It was concluded that the single arch MEAW technique combined with class III elastics can correct skeletal class III malocclusion. The MEAW technique and modified class III elastics provided an appropriate treatment strategy, especially for patients with a high angle and a tendency to open bite.
\end{abstract}

Keywords: MEAW technique, class III skeletal patter.

\section{Introducción}

De acuerdo con la Organización Mundial de la Salud (OMS); las maloclusiones ocupan el tercer lugar de prevalencia dentro de las patologías de salud bucodental luego de la caries dental y de la enfermedad periodontal. En nuestra región se presentan altos niveles de incidencia y prevalencia de maloclusiones que superan el $85 \%$ de la población(1).

Los individuos con maloclusión clase III pueden tener combinaciones de componentes esqueléticos y dentoalveolares en los tres planos del espacio: transversal, sagital y vertical $(2,3)$; por lo que se considera que es de etiología multifactorial. Dentro de estos factores se encuentran: causas congénitas o adquiridas, como síndromes; causas funcionales, como amigdalitis recurrentes; causas locales, entre las que se encuentran las interferencias oclusales, que son causantes de maloclusión clase III funcional; pérdida prematura de molares deciduos o agenesia de dientes maxilares que pueden estar relacionados con hipoplasia maxilar; presencia de dientes supernumerarios 
mandibulares que provocan aumento en el tamaño del arco mandibular $(2,4)$.

La maloclusión clase III es un fenotipo dentofacial heterogéneo, que se caracteriza por el crecimiento excesivo de la mandíbula; una deficiencia del maxilar, o bien una combinación de ambos, y puede ocurrir, ya sea como parte de un síndrome, o de manera aislada. La maloclusión clase III, generalmente, se manifiesta desde una edad muy temprana, y típicamente se evidencia por una relación incisal borde a borde, o por una mordida cruzada anterior (5).

Existen muchas alternativas de tratamiento para camuflar este tipo de maloclusión con patrón esquelético de clase III. Una alternativa es el tratamiento con extracciones de primeros premolares en el maxilar inferior $(6,7)$; así como la distalización de la dentición inferior $(8,9)$, los mismos que pueden camuflar esta maloclusión; sin embargo, podría llevarnos un tiempo más prolongado de tratamiento, así como causar algunas recidivas postratamiento. Una nueva alternativa surgida en los últimos años, y que ofrece buenos resultados y pocas complicaciones postratamiento, es la filosofía MEAW. Esta técnica comprende el uso de arcos multiansas; estos van colocados en los espacios interproximales de cada pieza dentaria desde distal del incisivo lateral hasta distal del primer molar, esto permite el control individual de cada pieza dentaria en los tres planos del espacio; asimismo esta técnica es usada en combinación de los elásticos intermaxilares. Los arcos MEAW son fabricados en alambre Elgiloy azul 0.016 x $0.022(10,11)$. No requiere de una prescripción específica de brackets. Esta técnica tiene como mecanismo de acción la rotación mandibular, a través de la modificación del plano oclusal con intrusión o extrusión de los molares para aplanar o empinar el plano oclusal, según requiera el caso a tratar (11).

\section{Caso clínico}

Paciente de sexo femenino, con 19 años y 8 meses de edad, de raza mestiza. Acudió con el motivo de la consulta: "Quiero corregir mis dientes".

Al realizar el examen clínico facial se observó un tipo dólicofacial, simétrico, con el tercio inferior aumentado, y con presencia de competencia labial, labios medianos y normotónicos. El análisis muestra una sonrisa asimétrica, de tipo baja. Presentaba el arco de la sonrisa no consonante y sin exposición gingival. Se observa también un perfil facial recto, hiperdivergente; los ángulos nasolabial y mentolabial aumentados (Fig. 1).

En el examen clínico intraoral se observó un riesgo bajo de caries y tejido gingival sin alteración; asimismo, presencia de una maloclusión con relaciones molares y caninas propias de la clase III; según la clasificación de Angle. La paciente presentaba mordida cruzada anterior, overbite negativo de $-2 \mathrm{~mm}(10 \%)$, overjet $2 \mathrm{~mm}$ y línea media superior e inferior alineada a la línea media facial (Fig. 2).

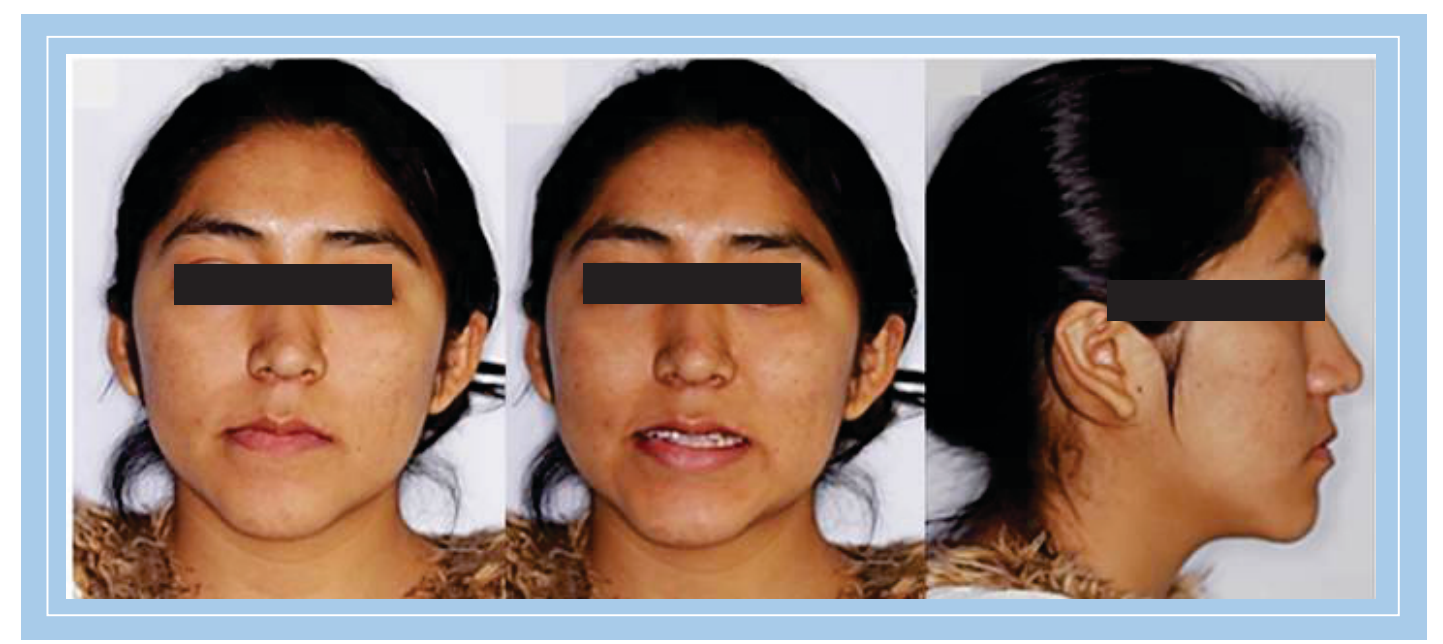

Figura 1. Fotografías iniciales de la paciente 


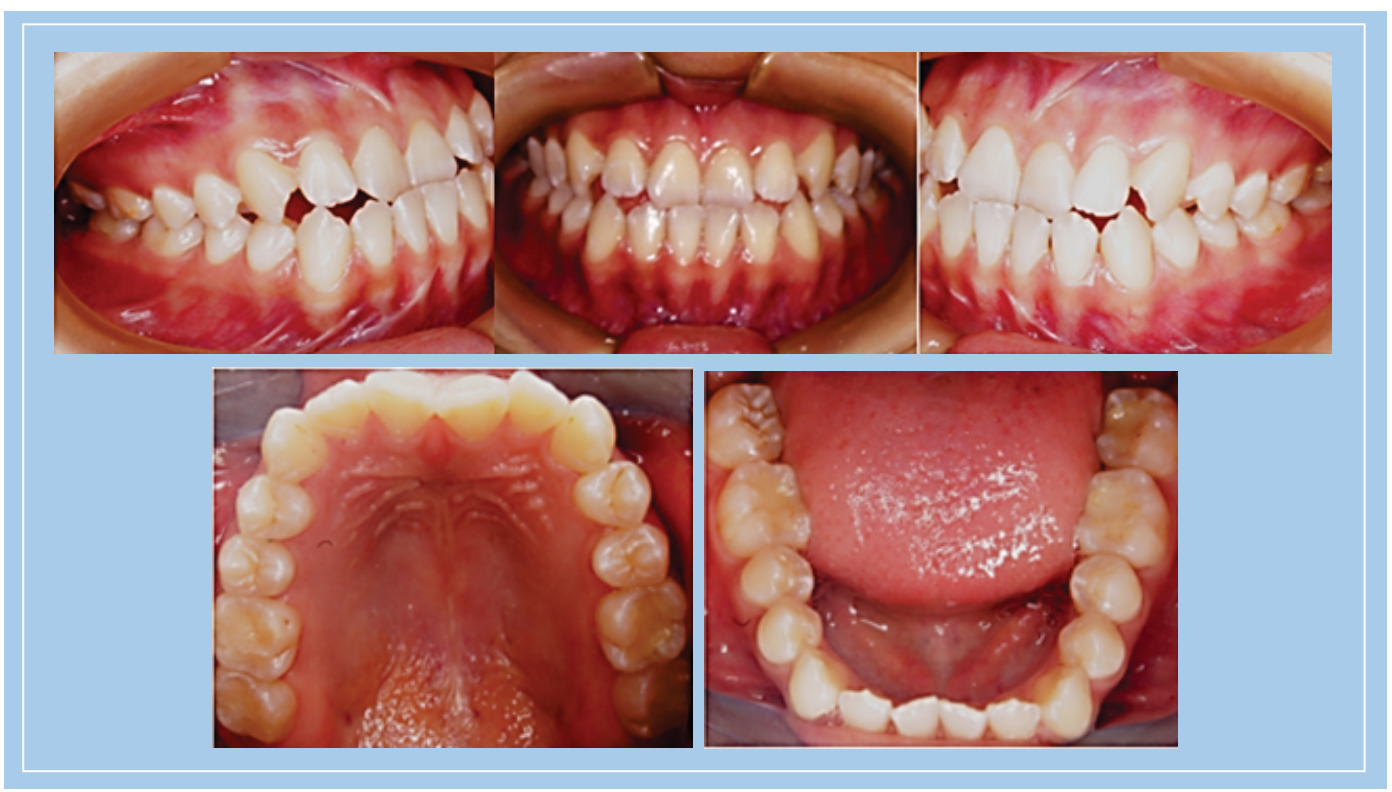

Figura 2. Fotografías intraorales iniciales.

En el examen radiográfico panorámico se puede observar ausencia de las piezas 38 y 48; asimismo, se observa raíces divergentes, rama y cuerpo mandibular elongado, y estructuras sin aparente alteración (Fig. 3) El trazado cefalométrico nos indica una relación esquelética de clase III $\left(\mathrm{ANB}=-1^{\circ}\right)$ con normoposición maxilar $\left(\mathrm{SNA}=82^{\circ}\right)$ y protrusión mandibular $\left(\mathrm{SNB}=83^{\circ}\right)$ y crecimiento vertical $\left(\mathrm{SNGoGn}=37^{\circ}\right)$; asimismo, presenta un plano oclusal empinado $(\mathrm{SNPO}=24)$. A nivel dentario se observa los incisivos superiores con protrusión (I-NA=6mm, I$\mathrm{NA}=25^{\circ}$ ); los incisivos inferiores se encuentran protruidos y proinclinados $\left(\mathrm{I}-\mathrm{NB}=7 \mathrm{~mm}, \mathrm{I}-\mathrm{NB}=28^{\circ}\right.$ ). El análisis de Kim nos indica la clase III esquelética $(\mathrm{APDI}=84)$ con tendencia a mordida abierta $(\mathrm{ODI}=52)$, aunque el paciente no requiera extracción $(\mathrm{CF}=136)$; el marco dental nos indica la discrepancia posterior $\left(\mathrm{A}^{\prime}-6^{\prime} / \mathrm{A}^{\prime}-\mathrm{P}^{\prime}=0.6\right)$. Finalmente, se observa que los molares se encuentran mesializados (ángulo intermolar $170^{\circ}$ ) (Fig. 4).

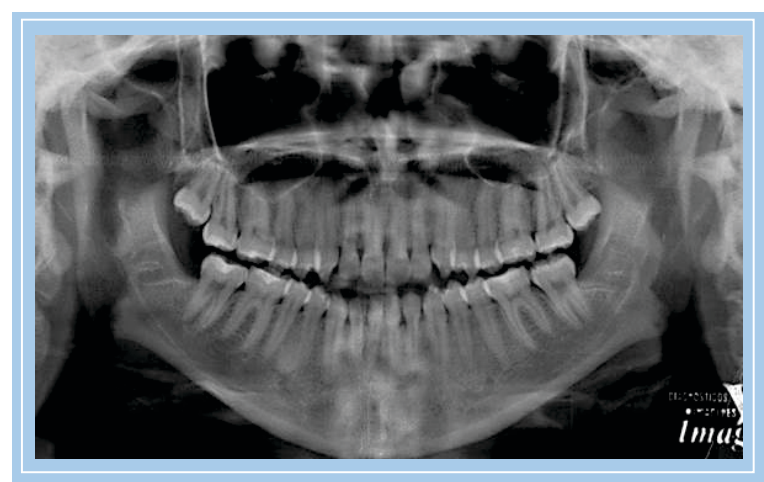

Figura 3. Radiografía panorámica.

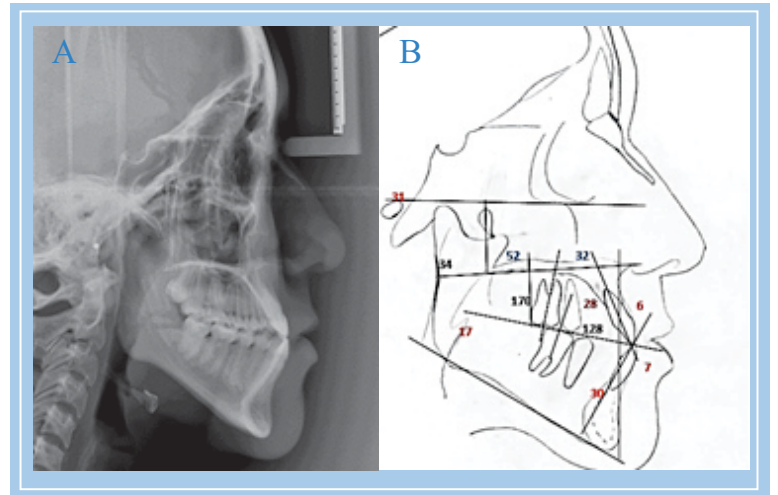

Figuras 4. A: Radiografía lateral inicial. B:

Cefalometría lateral inicial.Evolución de caso

Se inició el tratamiento con la instalación de la aparatología fija con brakets MBT slot 0.022"; asimismo se colocó arcos niti 0.014 superior y acero 0.012 inferior por un promedio de 4 meses, luego se procedió a la instalación de arco MEAW inferior en Elgiloy azul 0.016 x 0.022 y arco de acero 0.014 superior. Se realizó levante de mordida en el sector posterior, colocando topes de resina en la superficie oclusal de las pzas. 26 y 46. Se indicó el uso de elásticos intermaxilares $3 / 16$ de 6 onzas (Fig. 5). Finalmente, la paciente siguió las indicaciones por dos meses.

\section{Evolución de caso}

Se inició el tratamiento con la instalación de la aparatología fija con brakets MBT slot 0.022”; asimismo se colocó arcos niti 0.014 superior y acero 0.012 inferior por un promedio de 4 meses, luego se procedió a la instalación de arco MEAW inferior en 
Elgiloy azul 0.016 x 0.022 y arco de acero 0.014 superior. Se realizó levante de mordida en el sector posterior, colocando topes de resina en la superficie oclusal de las pzas. 26 y 46 . Se indicó el uso de elásticos intermaxilares 3/16 de 6 onzas (Fig. 5). Finalmente, la paciente siguió las indicaciones por dos meses.

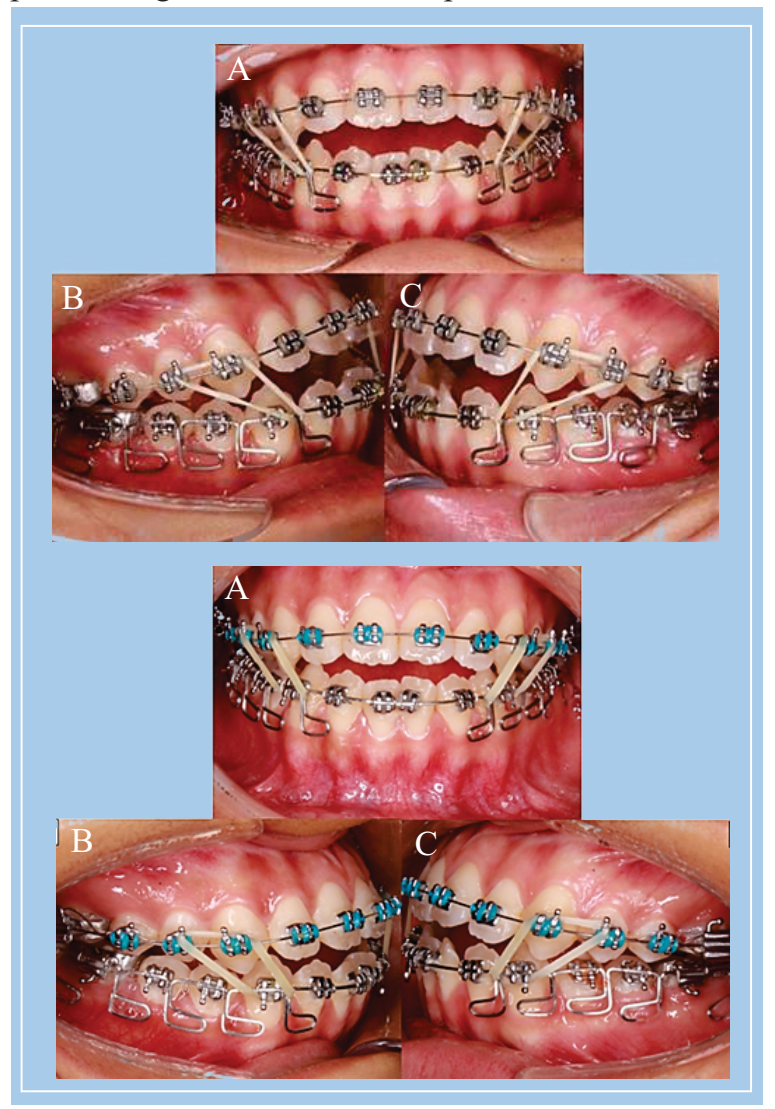

Figura 5. Elásticos cortos de clase III.

Después de que la paciente se ausentara por un periodo de 5 meses se reinició el tratamiento reactivando el arco MEAW inferior y arco de acero superior 0.014 (Fig. 6).

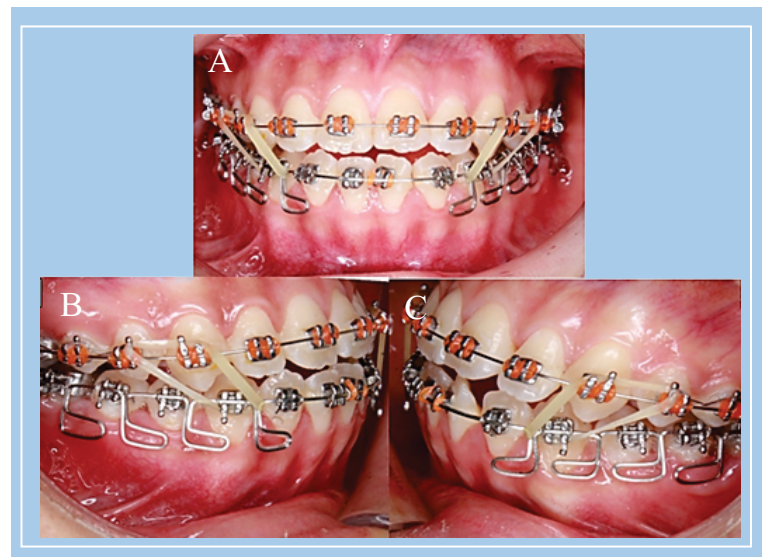

Figura 6. Elásticos cortos de clase III.
Luego de 3 meses de uso del Arco MEAW (con elásticos intermaxilares) se logró conseguir una clase I canina y molar, así como el descruce de la mordida anterior. Se retiró el arco MEAW, luego se cambió por arco niti 0.012. Asimismo, se indicó el uso de elásticos intermaxilares en clase I, luego se continuó con arcos 0.016 de acero superior e inferior.

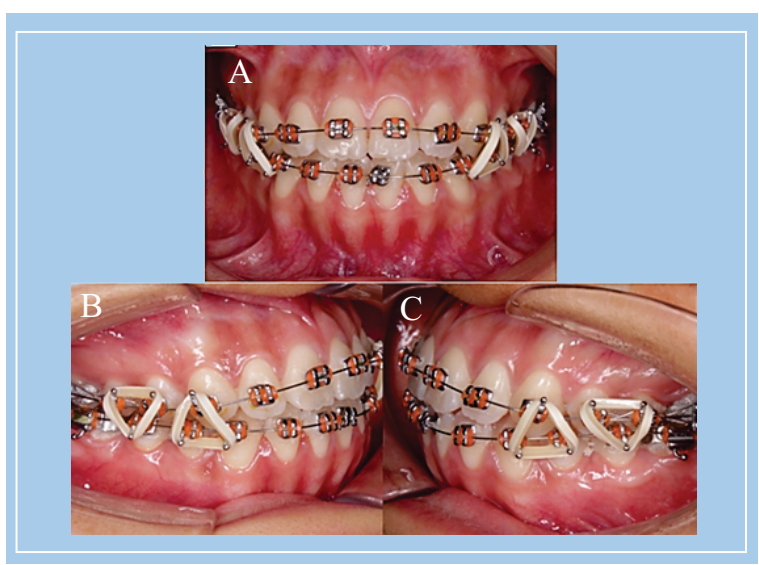

Figura 7. Elásticos de clase I.

Después de 6 meses se logró finalizar con los detalles faltantes para una buena intercuspidación. El término del tratamiento se realizó con arco niti 0.016 x 0.022 , superior e inferior; luego se realizó el amarre con ligadura de molar a molar en ambas arcadas; además se procedió a realizar la segmentación de arcos a nivel de canino y premolar (Fig. 8) con el fin de lograr una buena compensación dentoalveolar y un mejor manejo de planos oclusales.

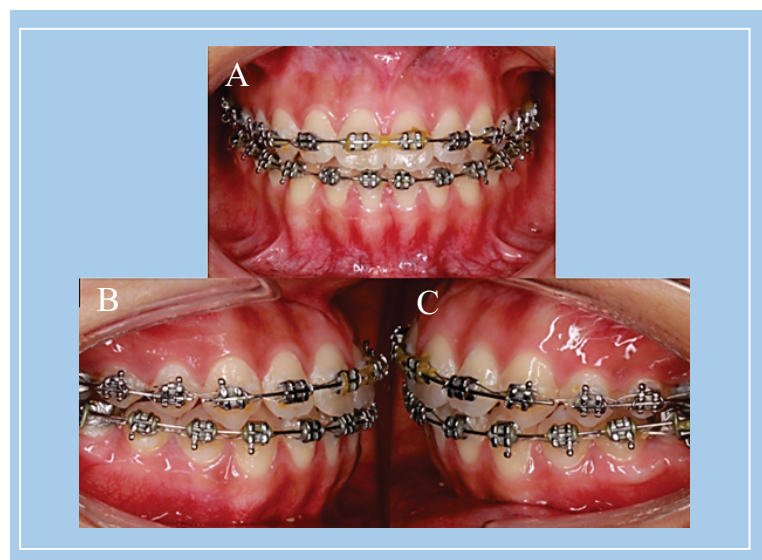

Figura 8. Segmentación de arcos.

Finalmente, 2 semanas después; habiendo conseguido la clase I canina y molar, un buen overbite y overjet, así como una buena guía canina e incisiva, y teniendo una intercuspidación óptima; se procedió a retirar los brackets y colocar una contención fija en el sector anteroinferior, y una contención removible en el arco superior (Fig. 9). 


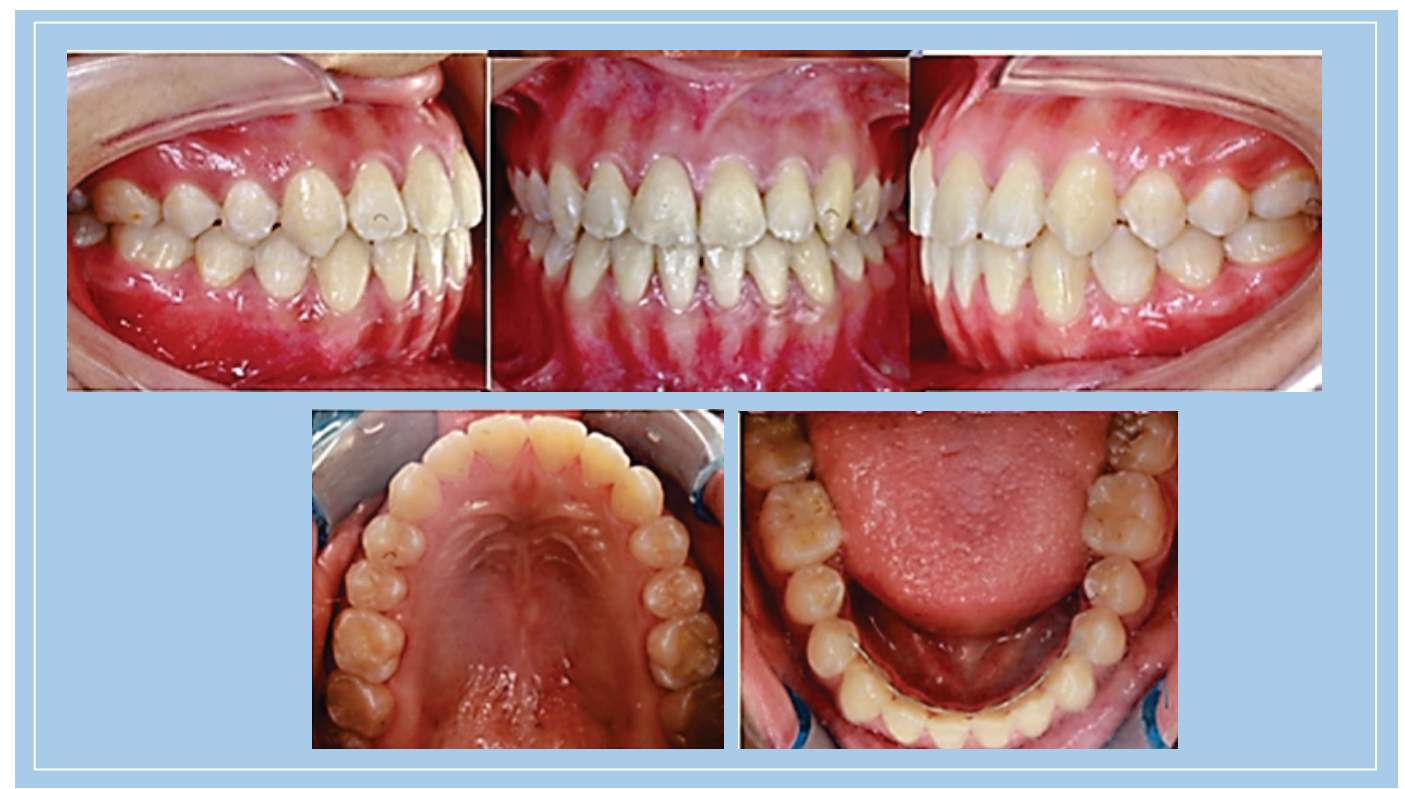

Figura 9. Fotografías intraorales, después del tratamiento.

Anivel del plano estético se logró la convexidad del perfil con adelantamiento del labio superior (Fig. 10).

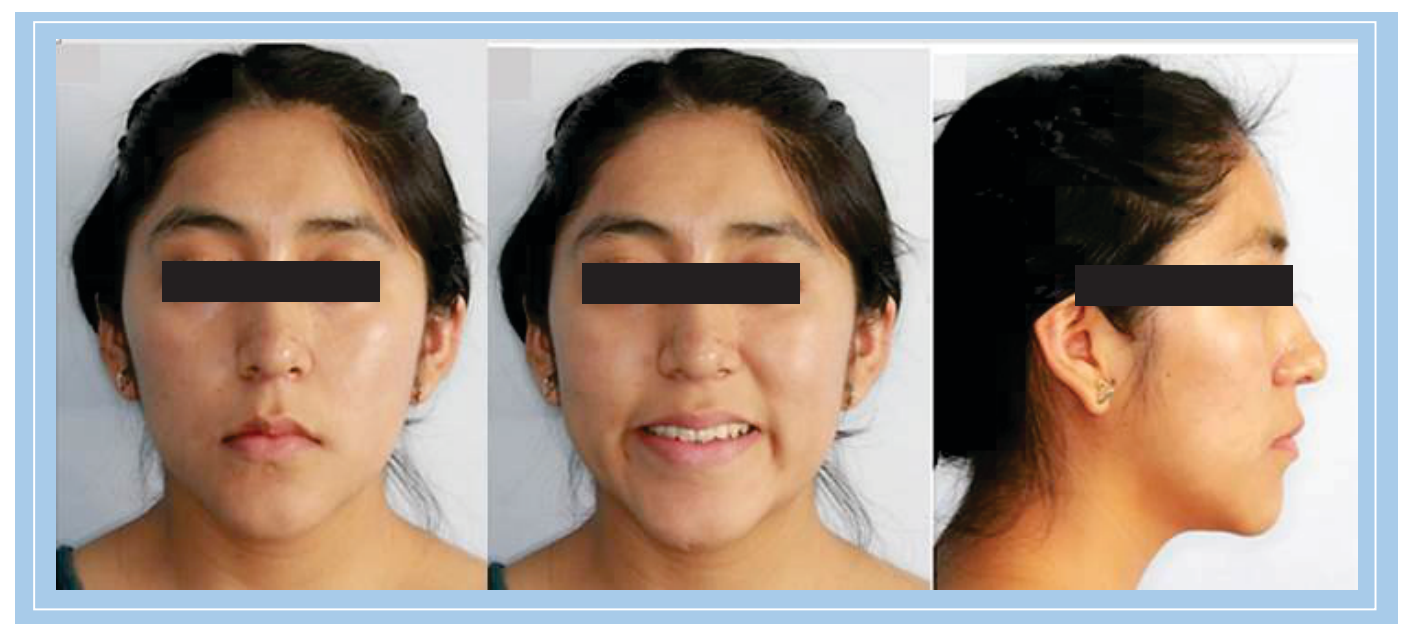

Figura 10. Fotografías extraorales, después del tratamiento.

En la cefalometría final podemos observar que se mantuvo la clase III esquelética $(\mathrm{ANB}=-1)$, normoposición de la mandíbula $(\mathrm{SNB}=79)$. A nivel dentario se logró un aplanamiento del plano oclusal $\left(\mathrm{SNGoGn}=20^{\circ}\right)$; asimismo existe una acentuación de la vestibularización en los incisivos ( $\mathrm{I}-\mathrm{NA}=14 \mathrm{~mm}$ ) (I$\mathrm{NB}=9 \mathrm{~mm})$; además se mejoró el ángulo intermolar $177^{\circ}$ (Fig. 11)

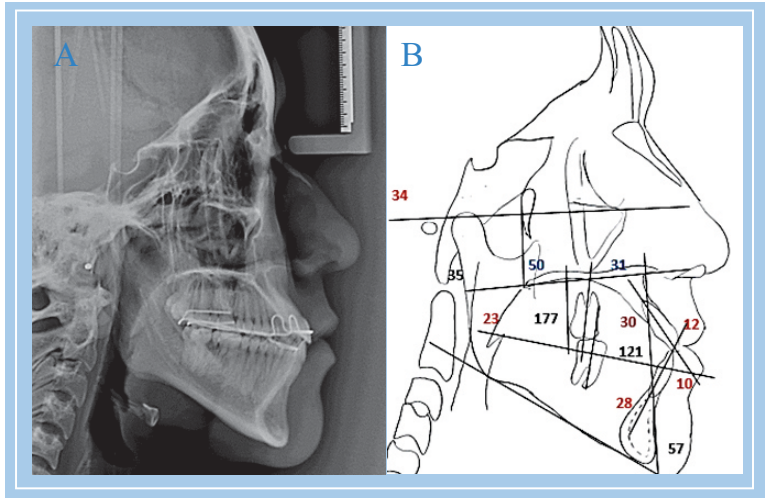

Figura 11. (A) Radiografía lateral final. (B) Cefalometría final. 
En la superposición de trazados podemos observar la extrusión del molar superior y la intrusión del molar inferior, así como la rotación posterior de mandíbula (Fig. 12).

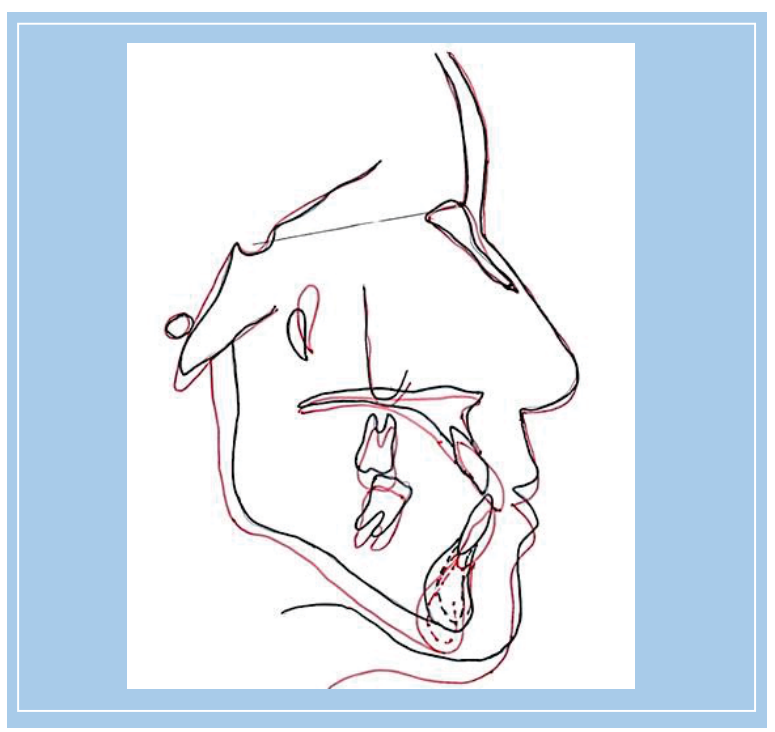

Figura 12. Trazado inicial (negro), trazado final (rojo).

\section{Discusión}

Shushu He et al. (12) demostraron que los tratamientos realizados con arcos MEAW y elásticos modificados con miniimplantes son muy eficaces logrando resultados en 14 y 18 meses respectivamente. En este trabajo también se utilizó arco MEAW con elásticos cortos de clase III, reafirmando la eficacia de la técnica MEAW.

Rincón (13) reportó un caso clínico tratado con arcos MEAW, superior e inferior, en combinación de elásticos intermaxilares de clase III con relación incisal borde a borde, cuyo tiempo de tratamiento fueron 8 meses. En este trabajo se usó un arco MEAW inferior con elásticos de clase III, con mordida cruzada anterior, cuyo tiempo efectivo de tratamiento es de 15 meses. La diferencia podría deberse a que en el caso anterior se resolvió una mordida borde a borde, mientras que el caso que aquí se presenta es una mordida cruzada anterior; razón por la cual lleva más tiempo resolver.

Yuichi Sakai et al. (14) reportaron un caso de mordida abierta, tratado con expansión rápida del maxilar, y el uso de miniplacas en la madíbula. El tiempo de tratamiento duró 23 meses. En este trabajo se realizó el camuflaje de clase III en una sola fase, y con el uso de arcos continuos y arco MEAW, logrando alcanzar nuestros objetivos en 15 meses efectivos de tratamiento. En comparación con el trabajo presentado por Yuichi et al. podemos inferir que, al ser el tratamiento con miniplacas, el costo es mayor en comparación del tratamiento con arcos MEAW.

Ypanaque (15) utilizó un arco MEAW inferior en combinación con un arco utilitario superior de protracción con striping inferior para aliviar el apiñamiento inferior en un tiempo de 26 meses. Sin embargo, en este trabajo se utilizó arco multiloop inferior en combinación de arcos continuos niti y de acero superiores redondos sin desgaste selectivo, logrando los objetivos deseados en un tiempo efectivo de 15 meses de tratamiento.

\section{Conclusiones}

El uso de los arcos MEAW son muy efectivos para el tratamiento compensatorio de los pacientes con patrón esquelético de Clase III, aunque requiere la colaboración del paciente en el uso de las ligas intermaxilares.

A nivel esquelético se logra una rotación posterior de la mandíbula, y esto corrige la protrusión mandibular.

A nivel dentario se obtiene un aplanamiento del plano oclusal con la extrusión del molar superior y la intrusión del molar inferior. Se consigue la clase I molar y canina con guía canina e incisiva, esto garantiza la estabilidad posterior al tratamiento.

El tratamiento mejora el perfil blando del paciente, esto ocasiona un impacto psicológico positivo.

Los efectos del tratamiento con arcos MEAW son muy positivos, los cuales se logran a través de la rotación mandibular y la modificación del plano oclusal; eliminando los efectos adversos frente a otras técnicas como las inclinaciones o rotaciones de los molares, logrando mayor estabilidad postratamiento en comparación con las técnicas de camuflaje con extracciones; asimismo el tiempo de tratamiento es más reducido. 


\section{Referencias bibliográficas}

1. Pérez GG, Fuenmayor LM. Mordida Cruzada anterior. Revisión Bibliográfica. Revista Latinoamericana de Ortodoncia y Odontopediatría. Disponible en : www.ortodoncia.ws/publicaciones/2012/pdf/art1 9.pdf

2. Escalona EE, Navarro MarBelR, Rivera HO, José M, Carreras L, Mora B, et al. Tratamiento temprano de las Clases III. RevEspOrtod. 2011; 41:79- 89.

3. Rabie ABM, Gu Y. Diagnostic criteria for pseudoClass III malocclusion. American Journal of Orthodontics and Dentofacial Orthopedics. 2000; 117(1):1-9.

4. Primozic J, Farcnik F, Perinetti G, Richmond S, Ovsenik M. The association of tongue posture with the dentoalveolar maxillary and mandibular morphology in Class III malocclusion: a controlled study. The European Journal of Orthodontics. 2013;35(3):388-93.

5. Singh GD. Morphologic determinants in the etiology of class III malocclusions: a review. Clin Anat 1999;12(5):382-405.

6. Alhaija EA, Alkateeb SN. Skeletal, dental and soft tissue changes in Class III patients treated with fixed appliances and lower premolar extractions. Australian orthodontic journal. 2011;27(1):41.

7. Ruelas $\mathrm{AC}$ de $\mathrm{O}$, Baratieri C, Roma MB, Izquierdo A de M, Boaventura L, Rodrigues CS, et al. Angle Class III malocclusion treated with mandibular first molar extractions. American Journal of Orthodontics and Dentofacial Orthopedics. 2012; 142(3):384-92.

8. Sobral MC, Habib FA de L, Matzenbacher L. Conservative compensatory Angle Class III malocclusion treatment. Dental Press Journal of
Orthodontics. 2012; 17(6):137-45.

9. Kuroda Y, Kuroda S, Alexander RG, Tanaka E. Adult Class 111 Treatment Using a J- Hook Headgear to the Mandibular Arch. The Angle Orthodontist.2009; 80(2):336-43.

10. Sadao S. MEAW, Orthodontic Therapy Using Multiloop Edgewise Archwire. kanagawa: Kanagawa Dental College; 2001.

11. Sadao S. Treatment approach to malocclusions under the consideration of craniofacial dynamics. Philippines: Grace Printing Press Inc.; 2001.

12. Shushu He, Jinhui Gao, Peter Wamalwa, Yunji Wang, Shujuan Zou, Song Chen; Tratamiento de camuflaje de la maloclusión esquelética de clase III con alambre de arco multiloop en el borde y elásticos de clase III modificados mediante anclajemaxilar de miniimplantes. Angle Orthod, 1 de julio de 2013;83(4):630-640.doi: https://doi.org/10.2319/091312-730.1

13. Rincón-Ducuara CL. Tratamiento de maloclusión de clase III con arco multiloop edgewise arch wire(meaw) reporte de caso clínico. CES Odont 2018;31(2): 76-93.

14. Yuichi Sakai, Shingo Kuroda, Sakhr A. Murshid, Teruko Takano-Yamamoto; Clase esquelética 111 Tratamiento de mordida abierta severa utilizando anclaje de implante. Angle Orthod, 1 de enero de $2008 ; 78$ (1):157-166.d o i : https://doi.org/10.2319/103006-445.1

15. Ypanaque Ramírez, Loidy Liceth. Tratamiento ortodóncico de camuflaje de una maloclusión de clase III. Trabajo Académico (Segunda Especialidad Profesional en Ortodoncia y Ortopedia Maxilar). Lima, Perú: Universidad Nacional Mayor de San Marcos, Facultad de Odontología, Unidad de Posgrado, 2018. 106 http://cybertesis.unmsm.edu.pe/handle/cybertesis 1936
Correspondencia:

deisyndeis@hotmail.com
Fecha de recepción : 15 de marzo de 2020

Fecha de aceptación : 15 de mayo de 2020 